\title{
ITER Core CXRS diagnostic: Assessment of different optical designs with respect to Neutronics criteria
}

Authors: ${ }^{1}$ B. Weinhorst, ${ }^{1}$ U. Fischer, ${ }^{2}$ G. Grossetti, ${ }^{3} \mathrm{Ph}$. Mertens, ${ }^{3} \mathrm{D}$. A. Castaño Bardawil

Karlsruhe Institute of Technology (KIT), Eggenstein-Leopoldshafen, Germany

${ }^{1}$ Institute for Neutron Physics and Reactor Technology (INR), ${ }^{2}$ Institute for Applied Materials (IAM)

Forschungszentrum Jülich $\mathrm{GmbH},{ }^{3}$ Institute of Energy and Climate Research (IEK-4 - Plasma Physics), 52425 Jülich, Germany

The Charge Exchange Recombination Spectroscopy (CXRS) diagnostic aims to measure emission lines of impurity isotopes in the ITER plasma in order to quantify several parameters like the composition of the plasma (density of helium, deuterium or tritium), the ion temperature or rotation velocities. The core plasma CXRS shall be installed in one of the ITER Upper Port Plugs (UPP \#3). Currently, four different optical layouts are being assessed with respect to the optical performance, engineering feasibility, cost, maintenance especially with respect to remote handling and the performance of their neutron radiation shielding.

This work is devoted to the neutronic analysis performed in support for the design of ITER CXRS-core Diagnostic System, presently under development by the IC3 Consortium (FZJ, KIT, BME, Wigner RCP, TU/Eindhoven, FOM-DIFFER, CCFE, CIEMAT, Optimal Optik).

In the paper, results of the neutronic analyses are presented showing the differences between the four different designs with respect to several nuclear responses such as neutron fluxes around the upper port plug and in the port interspace, maps of nuclear heating around the UPP including the toroidal/poloidal field coils as well as the vacuum vessel. Furthermore, radiation damage maps were calculated covering large areas of the upper port plug and of its environment. The results indicate the viability of one of the preferred designs from the neutronic point of view but also show the potential for improvements. 